\title{
Circular PVT1: an oncogenic non-coding RNA with emerging clinical importance
}

\author{
Jayashree Adhikary, ${ }^{1}$ Sourabrata Chakraborty, ${ }^{2}$ Subhamita Dalal, ${ }^{1}$ Souradip Basu, ${ }^{3}$ \\ Abhijit Dey, ${ }^{2}$ Amlan Ghosh $^{\oplus}{ }^{1}$
}

${ }^{1}$ Department of Life Sciences, Presidency University Kolkata, Kolkata, India

'Department of Life Sciences, Presidency University Kolkata, Kolkata, India

${ }^{3}$ Vidyasagar College, Kolkata, India

\section{Correspondence to} Dr Amlan Ghosh, Department of Life Sciences (Genetics of Non Communicable Disease), Presidency University Kolkata, Kolkata 700073, West Bengal, India; amlan.dbs@presiuniv. ac.in

JA and SC contributed equally.

Received 9 April 2019 Revised 14 May 2019

Accepted 15 May 2019 Published Online First

1 June 2019

\section{ABSTRACT}

The importance of circular RNAs (circRNAs) in pathological processes like cancer is evident. Among the circRNAs, recent studies have brought circPVT1 under focus as the most potent oncogenic non-coding RNA. Recent studies on various aspects of circPVT1, including its biogenesis, molecular alteration and its probable role in oncogenesis, have been conducted for research and clinical interest. In this review, a first attempt has been made to summarise the available data on circPVT1 from PubMed and other relevant databases with special emphasis on its role in development, progression and prognosis of various malignant conditions. CircPVT1 is derived from the same genetic locus encoding for long non-coding RNA IncPVT1; however, existing literature suggested circPVT1 and IncPVT1 are transcripted independently by different promoters. The interaction between circRNA and microRNA has been highlighted in majority of the few malignancies in which circPVT1 was studied. Besides its importance in diagnostic and prognostic procedures, circPVT1 seemed to have huge therapeutic potential as evident from differential drug response of cancer cell line as well as primary tumors depending on expression level of the candidate. circPVT1 in cancer therapeutics might be promising as a biomarker to make the existing treatment protocol more effective and also as potential target for designing novel therapeutic intervention.

\section{INTRODUCTION}

Non-coding RNAs (ncRNAs) comprise a major portion of the human genome ${ }^{1}$ and are considered under two broad heads: housekeeper ncRNAs, that is, ribosomal RNA (rRNA), transfer RNA (tRNA), small nuclear RNA (snRNA) etc. and regulatory ncRNAs. ${ }^{2}$ Regulatory ncRNAs of $<200$ base pairs (bp) are small ncRNAs like microRNA (miRNA), Piwi-interacting RNA (piRNA) etc. and those of $>200$ bp in length are long ncRNAs (lncRNAs). ${ }^{3}$ LncRNA may be sense, antisense, bidirectional, intronic or intergenic depending on their transcriptional origin and direction with respect to that of adjacent or host protein coding genes. ${ }^{2}$ Recently, circular RNAs (circRNAs) have emerged as another important class of lncRNAs. CircRNAs are covalently closed loop-like structure with no $5^{\prime}$ and $3^{\prime}$ polarity, and their biogenesis seemed to occur in a process different from canonical splicing of linear RNA; moreover, endogenous circRNA did not seem to be involved in ribosome-assisted translation indicating its different mode of function as a new class of ncRNA. ${ }^{4}$ HUMAN PVT1 gene (homologous to mouse plasmacytoma variant translocation-1) that encodes long non-coding RNA, IncPVT1, is located on chromosome $8 \mathrm{q} 24.21$ adjacent to $c-M y c{ }^{5}$ PVT1 gene showed frequent amplification at genome level and concordant transcriptional upregulation in different malignancies. ${ }^{67}$ Moreover, molecular alteration of lncPVT1 was significantly associated with poor patient outcome indicating it as an important oncogenic lncRNA with prognostic and therapeutic potentials. ${ }^{8-12}$ Recently, one circRNA derived from PVT1 gene was detected and named circPVT1 accordingly. ${ }^{13}$ Like lncPVT1, overexpression of circPVT1 and its prognostic importance in few malignancies was also reported. ${ }^{14}{ }^{15}$ Interestingly, circPVT1 seemed to be expressed independently of $\operatorname{lncPVT} 1^{15} 16$ and might affect a wide variety of molecular pathways that remained mostly uncharacterised. Considering the importance of circPVT1 in the emerging field of non-coding RNA and its potential association in disease pathogenesis that remained mostly unexplored, a review work summarising the published data would be helpful for any further research in this respect. Although few reviews on circRNA have been published recently, ${ }^{17-19}$ to our best knowledge, no one has discussed circPVT1 in detail.

Under aforesaid backdrop, this review attempts to summarise available data on circPVT1 including its biogenesis, reported alterations with associated molecular pathways in different malignancies and its potency as diagnostic and prognostic biomarkers, as well as target for novel therapeutic strategies.

\section{Genomic locus of circPVT1}

With respect to genomic locus, PVT1 is considered as the host gene of circPVT1 (figure 1). However, circPVT1 seems to be transcribed from its own promoter which is different from that of lncPVT1, indicating an independent mode of expression of the candidates. ${ }^{1516}$ HUMAN PVT1 is located on the long arm of chromosome $8(8 \mathrm{q} 24)$ that harbours a preferred site of genetic aberrations like translocation, ${ }^{20}$ amplification $^{5}$ and viral integration ${ }^{21}$ reported in a wide variety of cancers. The region also harbours multiple risk loci for different diseases other than malignancies ${ }^{22} 23$ as well as a 1.8 $\mathrm{Mb}$ region of gene desert ${ }^{24}$ that seems to exert some sort of control on transcription of distant genes and considered as risk site of different cancers. ${ }^{25} 26$ Moreover, chromosomal 8q24 harbours oncogene $c-M y c$, about $60 \mathrm{~Kb}$ upstream of PVT1 gene (human genome GRch38/hg38). Functional interaction of 


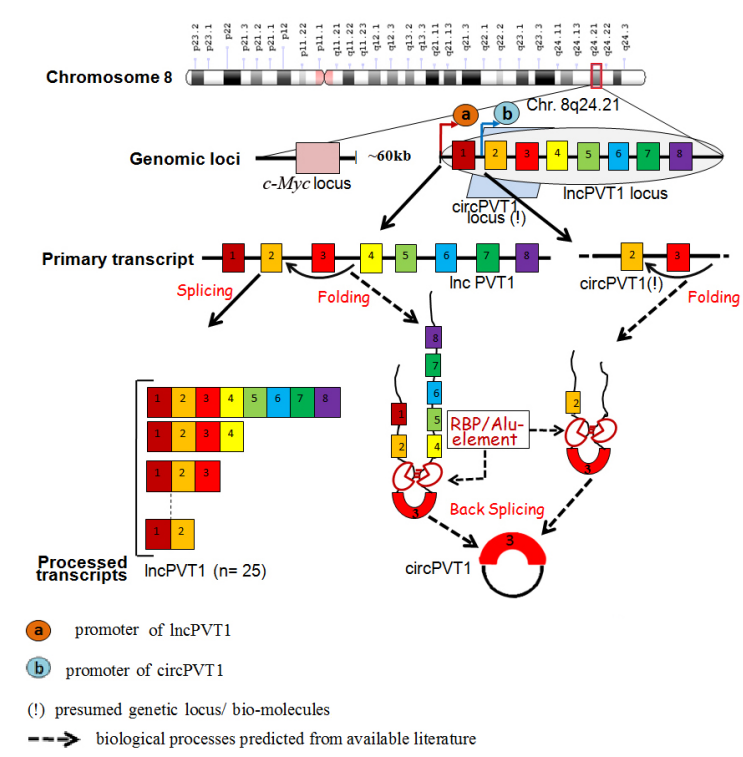

Figure 1 Schematic view of human genomic region harbouring c-MycIPVT1 loci and probable mode of biogenesis of circPVT1 with respect to that of IncPVT1. 1-8, exons of PVT1 locus; circPVT1, circular PVT1; IncPVT1, long non-coding RNA PVT1; RBP, RNA binding protein.

c-Myc and lncPVT1 has been reported previously, ${ }^{27}$ and now such interaction with circPVT1 is also evident. ${ }^{28}$

\section{BIOGENESIS}

\section{Biogenesis of circRNA}

CircRNAs may vary with respect to genomic constituent and are categorised accordingly into circular intronic RNAs (ciRNAs), ${ }^{29}$ exonic circRNAs (ecircRNAs) ${ }^{30}$ and exonic-intronic circRNAs (ELciRNAs). ${ }^{31}$ CircRNAs were previously considered as mis-spliced product of linear RNA $\mathrm{RN}^{32}$ or an intermediate of intronlariat. ${ }^{33}$ But recent studies considered circRNA as a product of back splicing or as stabilised intron escaped from de-branching, and proposed models in this respect were exon skipping, ${ }^{34} 35$ direct back splicing, ${ }^{34} 35$ alternative circularisation, ${ }^{36}$ competitive RNA pairing ${ }^{37}$ and RNA binding protein (RBP)-mediated circularisation. $^{38}$

In contrast to classical canonical splicing, back splicing joins a downstream splice donor site $\left(3^{\prime}\right)$ to an upstream splice acceptor site $\left(5^{\prime}\right)$ in reverse orientation to form a covalently closed circRNA. ${ }^{39}$ Available literature suggested back splicing as a phenomenon coupled with classical canonical splicing as in the former, both canonical splice signals and canonical spliceosomal machinery were used..$^{370}$ As per available data, both linear and circular RNA were derived from a single pre-mRNA transcript that might undergo back splicing first followed by canonical splicing or vice versa; accordingly, circRNA might be formed directly from the pre-mRNA transcript (direct back splicing) or indirectly from a lariat intermediate derived from canonical splicing of the pre-mRNA. ${ }^{3541}$

However, in back splicing, circularisation of exons or exonintron structures did not seem to be favoured by canonical spliceosome machinery. Some cis (Alu element, non-repetitive complementary sequences) and trans acting factors (RBP) were proposed to mediate pairing across the flanking introns and thus bringing downstream donor site close to upstream acceptor sites to promote back splicing. ${ }^{3038}$

\section{Biogenesis of circPVT1}

Biogenesis of circPVT1 remained poorly understood until the present. CircPVT1 was first detected in gastric cancer and found to contain exon 3 with flanking introns of lncPVT1 in circularised form. ${ }^{13}{ }^{14}$ Although lncPVT1 expressed as many as 25 transcript variants in humans of which exon 3 is skipped in some variants, there was no direct evidence for a common precursor of both circPVT1 and lncPVT1. According to available data in the published literature, circPVT1 seemed to express independently from a separate promoter upstream of exon 2 of its host gene. ${ }^{15}$ Therefore, circPVT1 and lncPVT1 might be generated from separate primary transcripts derived from their own promoters (figure 1). Similarly, Ghosh $e t a l^{42}$ and Mitra $e t a l^{43}$ suggested independent mode of transcription of two lncRNAs (BC017743 and $\mathrm{BC} 043430)$ from separate promoters with respect to that of the host gene ROBO1/DUTT1 in head and neck squamous cell carcinoma and cervical carcinoma, respectively. Furthermore, circPVT1 seemed to control its own expression in a positive feedback manner in association with mutant p53 and some candidate proteins of Hippo pathway like YAP, TEAD and so on. ${ }^{15}$ Actually, circPVT1 was reported to interact with YAP, mutant p53 and TEAD in a complex and then was recruited to its own promoter for transcriptional upregulation. Furthermore, mutant p53-mediated enhanced transcription of circPVT1 was found to coincide with reduced expression of lncPVT1 at primary transcript level; therefore, in presence of mutant $\mathrm{p} 53$, transcriptional machinery seemed to be recruited preferentially to the promoter of circPVT1 than that of lncPVT1. The exact mechanism of generation of circPVT1 from its primary RNA transcript has not been studied yet and the role of associated cis and/or trans acting factors has not been characterised. However, lncPVT1 was reported to bind with a number of $\mathrm{RBPs}^{44}$ and some of the binding sites reside within the flanking introns of exon 3, the genomic region reported to encode circPVT1. Moreover, IncPVT1 was enriched in repetitive Alu-like elements that seemed to play a crucial role in its nuclear retention. ${ }^{45}$ These findings raised the possibility of both repetitive elements and RBPs to play an important role as cis and trans acting factors, respectively, in back splicing of circPVT1 from its precursor (figure 1).

\section{DETECTION}

\section{Detection of circRNA}

Though the first circRNA was identified way back in the $1980 \mathrm{~s},{ }^{46}$ knowledge in this respect remained limited for a long time. circRNAs do not have any free $3^{\prime}$ or $5^{\prime}$ end and hence not suitable to be analysed by traditional methods like size or electrophoretic mobility shift assay, rapid amplification of cDNA ends (RACE) and so on. However, very recently, few modern approaches have been devised for detection and characterisation of circRNA.

Sequence-based approaches are used recently for genomewide studies of circRNA. In this method, reads from RNA-seq data are used and aligned to all possible candidate exon junctions for detection of circRNA. ${ }^{41}$ Alternatively, RNA-seq data may be matched to genomic sequences first and then unmapped reads subjected to further analysis by different algorithms to identify back-spliced junction. ${ }^{14}$ However, circRNAs have overlapping sequences with their linear counterparts and the former category seems to be less abundant than the latter. Enrichment of circular template in total RNA content by different methodologies has been recommended to overcome these limitations. circRNAs, as lacking any linear ends, are resistant to exonuclease activity 
of RNase R. Therefore, total RNA content may be subjected to RNase R treatment to enrich circular template. ${ }^{47}$ However, after RNase R treatment, a considerable fraction of linear RNA might remain undigested or partially digested, and to remove this for maximising the circular template, Panda $\mathrm{et} \mathrm{al}^{48}$ introduced a new device, RPAD (RNase R treatment followed by Polyadenylation and poly(A)+RNA Depletion). In RPAD, RNase R-resistant linear RNAs are subjected to poly(A) tailing enzymatically and then removed by using oligo(dt) beads to get samples with highly concentrated circular template; moreover, in this technique apart from mRNA, small RNAs like snRNAs, miRNAs and so on can also be removed effectively from the sample mixture. Recently, analysis of rRNA-depleted (ribo minus) or rRNA-depleted and non-poly(A) RNAs sequence data is recommended in sequence-based approaches. This method is devised to eliminate majority of reads of linear RNA to enrich back-spliced reads and therefore seemed to be more suitable for detection of poorly expressed circRNA. ${ }^{49}$

Divergent reverse transcriptase (RT)-PCR followed by Sanger sequencing and northern blot are frequently used for experimental validation of circRNAs. ${ }^{1450}$ Before PCR or northern blot, sample mixture may be treated with RNase R for enrichment of circular template. Primers are designed to flank the backspliced junction and are away from each other (divergent), so linear transcripts have no amplification; but the same primer set becomes convergent when the transcript gets circularised, so amplification occurs. This ensures specific PCR product from the circular template only.

CircInteractome (http://circinteractome.nia.nih.gov/), a database providing information of interacting miRNAs and RNA binding proteins of circRNAs, can be used to design divergent primers. ${ }^{51}$ In northern blot, probes encompassing exonic sequence of circRNA will detect both circRNA and its linear counterpart; however, if denatured gel is used instead of a native one, circRNA and linear RNA with the same nucleotide length can be distinguished by circRNA's slower migration rate. ${ }^{52}$

\section{Detection of circPVT1}

CircPVT1 was first detected in gastric cancer tissue by Chen et al. ${ }^{14}$ Briefly, ribo minus RNA seq data were generated from total RNA extract of target tissue and unmapped reads were subjected to computational algorithm (anchor alignment) to identify circRNAs. Divergent RT-PCR followed by Sanger sequencing was done for validation of circPVT1, and furthermore the candidate was found to be more stable and preferentially more cytoplasmic in localisation compared with its linear

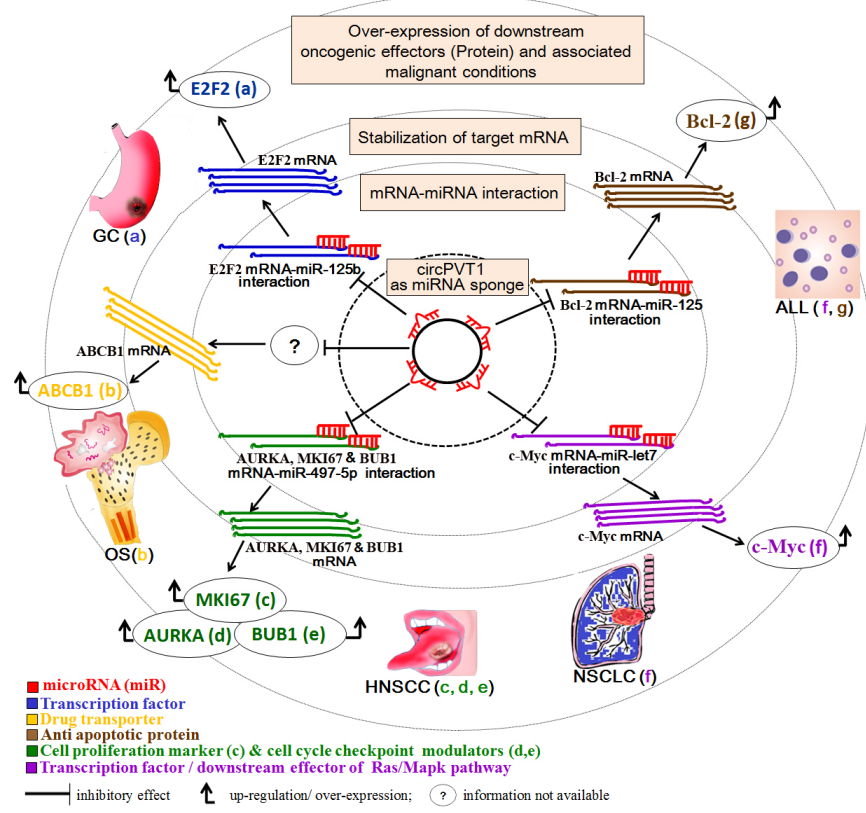

Figure 2 Summarised view on mode of oncogenesis of circPVT1 and associated malignancies of different organs. ALL, acute lymphoblastic leukaemia; GC, gastric carcinoma; HNSCC, head and neck squamous cell carcinoma; NSCLC, non-small cell lung carcinoma; OS, osteosarcoma.

counterpart lncPVT1. In head and neck squamous cell carcinoma (HNSCC), Verduci et $a l^{15}$ identified circPVT1 by quantitative RT-PCR followed by Sanger sequencing. However, in HNSCC cell line, circPVT1 was also detected in nucleus in addition to cytoplasm. In acute lymphoblastic leukaemia, osteosarcoma and non-small cell lung cancer, Hu et al, ${ }^{28}$ Kun-Peng $e t a l^{53}$ and Qin et $a l^{54}$ also used quantitative RT-PCR for detection of circPVT1.

\section{REPORTED ALTERATION OF CIRCPVT1 IN CANCER}

Importance of circRNA in pathogenesis of malignant condition has been reported recently. With advancement of circRNA research, expression of different circRNAs was detected in normal and diseased tissue of which circPVT1 seemed to be a potential candidate of oncogenic interest. As per available literature, so far circPVT1 has been studied in gastric cancer, HNSCC, acute lymphoblastic leukaemia, osteosarcoma and non-small cell lung carcinoma (table 1; figure 2).

Table 1 Summarised information on role of circPVT1 in malignancy of different organs

\begin{tabular}{|c|c|c|c|c|c|c|c|}
\hline Circular RNA & Disease (cancer) & Molecular alteration & Functional role & Target miRNA & $\begin{array}{l}\text { Downstream } \\
\text { oncogenic effector }\end{array}$ & $\begin{array}{l}\text { Clinical } \\
\text { importance }\end{array}$ & Reference \\
\hline \multirow[t]{5}{*}{ Circular PVT1 } & Gastric cancer & $\begin{array}{l}\text { Genomic amplification } \\
\text { Overexpression }\end{array}$ & Proliferation ( $\uparrow)$ & miR-125b & $E 2 F 2$ & Prognostic & Chen et $a l^{14}$ \\
\hline & HNSCC & Overexpression & $\begin{array}{l}\text { Proliferation ( } \uparrow) \\
\text { Migration }(-)\end{array}$ & miR-497-5p & $\begin{array}{l}\text { AURKA, MKI67 and } \\
\text { BUB1 }\end{array}$ & $\begin{array}{l}\text { Prognostic } \\
\text { Therapeutic }\end{array}$ & Verduci et $a l^{15}$ \\
\hline & $\begin{array}{l}\text { Acute lymphoblastic } \\
\text { leukaemia }\end{array}$ & Overexpression & $\begin{array}{l}\text { Proliferation ( } \uparrow) \\
\text { Apoptosis }(\downarrow)\end{array}$ & miR-125, miR-let7 & $c-M y c, B c l-2$ & Therapeutic & Hu et $a l^{28}$ \\
\hline & Osteosarcoma & Overexpression & $\begin{array}{l}\text { Proliferation }(\uparrow) \\
\text { Chemoresistance }(\uparrow)\end{array}$ & Unknown & $A B C B 1$ & $\begin{array}{l}\text { Diagnostic } \\
\text { Prognostic } \\
\text { Therapeutic }\end{array}$ & Kun-Peng et $\left.a\right|^{53}$ \\
\hline & NSCLC & Overexpression & $\begin{array}{l}\text { Proliferation ( } \uparrow) \\
\text { Apoptosis }(\downarrow)\end{array}$ & miR-497 & $B C l-2$ & $\begin{array}{l}\text { Prognostic } \\
\text { Therapeutic }\end{array}$ & Qin et $a l^{54}$ \\
\hline
\end{tabular}

$\uparrow$, positive impact; $\downarrow$, negative impact; - , no effect.

HNSCC, head and neck squamous cell carcinoma; NSCLC, non-small cell lung carcinoma. 


\section{Gastric cancer}

CircPVT1 was first identified as the most differentially expressed circRNA in gastric cancer (GC) by Chen et al. ${ }^{14}$ Overexpression of circPVT1 was found in majority of the GC tissues compared with matched non-cancerous part, and amplification of genomic region encoding for the candidate was suggested as the underlying mechanism. The authors also found significant transcriptional upregulation of IncPVT1 in GC, but interestingly this phenomenon was not coincided with expression of circPVT1; it indicated independent transcriptional or post-transcriptional regulation of the candidates in GC tissue. SiRNA (small interfering RNA)-mediated knockdown of circPVT1 was found to suppress proliferation of GC cells even in the presence of normal level of lncPVT1. This finding was further validated in colony formation and 5-ethynyl-2'-deoxyuridine (Edu) incorporation assay and it seemed to impose more importance on circPVT1 over its linear counterpart in tumorigenesis of gastric tissue. On the contrary, Huang et al, ${ }^{55} \mathrm{Xu}$ et $a l^{16}$ and Zhao et al ${ }^{56}$ reported significant positive influence of lncPVT1 on proliferation, invasion, growth and angiogenesis of GC in vitro and in vivo. Like other circRNAs, circPVT1 also served as a miRNA sponge and in GC, experimental evidence showed mir-125b as its most potential target. Actually, circPVT1 was proposed to bind and attenuate the tumor suppressive activity of miR-125b, and this seemed to facilitate overexpression of its target E2F2 transcription factor leading to uncontrolled cell proliferation and tumorigenesis. Like circPVT1, lncPVT1 might serve as miRNA sponge and $\operatorname{miR} 152^{57}$ and miR $186^{55}$ were reported as its downstream targets in GC leading to upregulation of oncogenic effectors like CD151, FGF2, HIF $\alpha$ and so on and tumor growth. Therefore, interpretation of Chen et $a l^{14}$ of imposing more importance on circPVT1 over its linear counterpart in pathogenesis of GC needs further experimental support for validation.

\section{Head and neck squamous cell carcinoma}

In HNSCC, Verduci et $a l^{15}$ found overexpression of circPVT1 and also its significant association with mutant Tp53 in the dataset of Cancer Genome Atlas. Later, this finding was validated in independent sets of HNSCC samples expressing mutant and wild-type TP53 proteins. In HNSCC cell line, siRNA-mediated knockdown of circPVT1 resulted in a significant decrease of cell proliferation; however, cell migration potential remained unaffected. Surprisingly, neither cell proliferation nor cell migration was affected significantly when only lncPVT1 was knocked down. However, in a recent study, Yu et $a l^{58}$ reported significant inhibition of cell proliferation, migration and invasion of HNSCC cell line after shRNA (short hairpin RNA)-mediated knockdown of lncPVT1. Although the investigators did not analyse expression of circPVT1 and mutant p53, two shRNAs (sh1: 5'-CCC AAC AGG AGG ACA GCT T-3' and sh2: 5'-GCU UGG AGG CUG AGG AGT T-3') used in the study were specific for exons 6 and 8 of PVT1 gene and hence did not seem to affect the expression of circPVT1; therefore, this might be a contrasting one with respect to findings reported by Verduci et al. ${ }^{15}$ From a panel of miRNAs showing significant downregulation in the presence of mutant p53, miR-497-5p was identified as the binding target of circPVT1 in HNSCC. ${ }^{15}$ miR-497-5p was known to have tumor-suppressive function and in HNSCC experimental evidence suggested three candidate genes, that is, AURKA, MKI67 and BUB1, as its potential target. AURKA encodes a serine-threonine kinase (Aurora A kinase) and MKI67 encodes the antigen KI67 that is associated with cellular proliferation and overexpression of the candidates has been found in HNSCC. ${ }^{59} 60$
$B U B 1$ encodes a protein kinase associated with spindle checkpoint and loss of function mutation or reduced expression of the candidate has been reported frequently in malignancy of different organs. ${ }^{6162}$ Therefore, in HNSCC, the role of AURKA and MKI67 as downstream oncogenic effectors of circPVT1 as proposed by Verduci et $a l^{15}$ was in accordance to existing literature; however, the candidature of BUB1 in this respect seemed to be a matter of scientific debate.

\section{Acute lymphoblastic leukaemia}

Consistent to the recent findings in gastric cancer and HNSCC, $\mathrm{Hu}$ et $a l^{28}$ reported significant overexpression of circPVT1 in acute lymphoblastic leukaemia (ALL) compared with normal bone marrow cells. Surprisingly, the authors did not find any change in expression of lncPVT1 and furthermore no significant correlation of this phenomenon was found with that of circPVT1 in their studied samples. shRNA-mediated knockdown of circPVT1 was found to reduce cell proliferation and induce apoptosis in B-ALL and T-ALL cell lines; furthermore, marked decrease of $\mathrm{c}-\mathrm{Myc}$ and $\mathrm{Bcl}-2$ at protein level as evident in knockdown experiment was suggested as the underlying mechanism. circPVT1 served as miRNA sponge and miR-125 $5^{14}$ and $\operatorname{miR}-l e t 7^{13}$ were considered as its potential binding target. miR-let7-mediated downregulation of c-Myc in nasopharyngeal carcinoma and mir-125b-mediated suppression of Bcl-2 expression in breast cancer were reported by Wong et $a l^{63}$ and Zhou et $a l,{ }^{64}$ respectively. Overexpression of c-Myc and $\mathrm{Bcl}-2$ was reported frequently in ALL and circPVT1-mediated downregulation of miR-125, and miR-let7 was proposed as the underlying molecular mechanism for this by $\mathrm{Hu}$ et al. ${ }^{28}$ However, in ALL, expression status of miR-125 and miR-let7 was explored in very few studies of which Mi et $a l^{65}$ reported reduced expression of miR-let7b in one, although Tassano et $a l^{66}$ found significant upregulation of miR-125b-1. Therefore, a substantial number of studies including different categories of ALL are warranted in this respect for better understanding of the issue in more details.

\section{Non-small cell lung carcinoma}

Qin et $a l^{54}$ found significant upregulation of circPVT1 in primary tumor and cell lines of non-small cell lung carcinoma (NSCLC). Significant suppression of cell proliferation and increase of apoptosis were also found in NSCLC cell line following shRNA-mediated knockdown of circPVT1. Furthermore, in circPVT1 knockdown cell, significant increase of $\mathrm{Bax}$ and decrease of $\mathrm{Bcl}-2$ proteins were evident and suggested as the underlying mechanism for suppression of oncogenic phenotypes. Like circPVT1, overexpression of lncPVT1 was reported frequently in NSCLC and suppression of proliferation, migration and invasion of NSCLC cell line was found after siRNA-mediated knockdown of the RNA. ${ }^{12}{ }^{67}$ However, in NSCLC, Qin et $a l^{54}$ did not analyse the expression of lncPVT1 or knock down the RNA for validation of oncogenic insult exclusive to circPVT1. In NSCLC, circPVT1 was reported to act as competing endogenous RNA of miR497 that was found to bind and attenuate Bcl-2 mRNA. However, miR497 was frequently downregulated in NSCLC ${ }^{68}$ and 1ncPVT1 was considered as the most potential regulator of such phenomenon. ${ }^{69}$ Thus, as per available literature, both lncPVT1 and circPVT1 was shown to undergo similar type of molecular alterations in NSCLC and both the candidates seemed to exert an oncogenic effect through common downstream effector by a similar mechanism. Therefore, detailed 
molecular analysis including both lncPVT1 and circPVT1 in the same set of sample is warranted to understand the oncogenic effect exclusive to each candidate in tumorigenesis of lung.

\section{Osteosarcoma}

Kun-Peng et $a l^{53}$ reported significant overexpression of circPVT1 in osteosarcoma (OS) compared with adjacent normal tissue. Oncogenic potential of circPVT1 was evident from reduced proliferation and increased drug sensitivity after siRNA-mediated knockdown of the RNA in OS cell line. In OS, IncPVT1 was also seen to enhance cellular proliferation, invasion as well as resistance to chemotherapeutic drugs, and miR-195, miR-497 and miR-152 were found to be immediate downstream targets of the lncRNA. ${ }^{8} 7071$ ATP-binding cassette subfamily B member 1 $(\mathrm{ABCB} 1)$ is a multidrug-resistant (MDR) protein involved in effluxing xenobiotics like drugs, toxins and so on, and its overexpression confers multidrug resistance in malignant tumors including osteosarcoma. ${ }^{72}$ In MDR cancer cell, miR-497-mediated attenuation of $\mathrm{ABCB} 1^{73}$ and miR-152 mediated downregulation of c-MET/PI3K/AKT pathway that seemed to upregulate ABCB1 expression ${ }^{71}{ }^{74}$ might serve as a possible molecular link of lncPVT1 with the drug transporter (ABCB1) and associated drug resistance phenomenon. ABCB1 was also considered as downstream oncogenic effector of circPVT1 in $\mathrm{OS}^{53}$; however, the underlying molecular mechanism was not explored.

\section{CIRCPVT1 AS POTENTIAL CLINICAL BIOMARKER}

Although studies on circPVT1 in malignancy remained scanty, significant association of the candidate with disease onset and outcome indicated it as a promising biomarker of diagnostic and prognostic importance. Some studies also suggested potential of circPVT1 to be explored for therapeutic purposes including improvement of existing treatment plan as well as designing novel intervention (table 1).

\section{Gastric cancer}

In GC, surprisingly significant inverse association of circPVT1 level was found with cases of advanced clinical stages and positive neural invasion; consistently poor overall and disease-free survival was evident in patients showing low level of circPVT1 in tumor. ${ }^{14}$ The authors failed to establish circPVT1 as an independent prognostic biomarker due to its low sensitivity and specificity in ROC (receiver operating characteristic) curve analysis. However, expression of lncPVT1 remained positively and significantly associated with poor patient outcome. ${ }^{14}$ Therefore, in this study, a combined score of cirPVT1 along with lncPVT1 was suggested as a better alternative for prediction of disease prognosis.

\section{Head and neck squamous cell carcinoma}

In HNSCC, significant poor survival of the patients showing overexpression of circPVT1 was reported and interestingly this phenomenon was found to be dependent on mutation of TP53. ${ }^{15}$ Cisplatin is a chemotherapeutic agent frequently used to treat HNSCC. siRNA mediated knockdown of circPVT1 was shown to increase sensitivity of HNSCC cell line to cisplatin with significant decrease of half maximum effective concentration (EC50) and half lethal concentration (LC50). ${ }^{15}$ Therefore, in HNSCC, optimum dose of cisplatin seemed to vary from one individual patient to another and circPVT1 level in tumor might be important in dose prediction for maximising the efficacy of treatment protocol.

\section{Acute lymphoblastic leukaemia}

CircPVT1 was significantly upregulated in ALL than that of healthy controls; however, the candidate did not seem to have enough sensitivity and specificity for diagnostic purpose as evident in ROC curve analysis. ${ }^{28}$ Overexpression of c-Myc and Bcl-2 accelerates cell division and prevents apoptosis during tumorigenesis. Experimental evidence suggested circPVT1 as the most potential target of novel therapeutics that might downregulate c-Myc and Bcl-2 selectively in ALL cells to induce apoptotic death. ${ }^{28}$

\section{Non-small cell lung carcinoma}

In NSCLC, significant association of circPVT1 was found with adverse clinicopathological features like tumor size, TNM stage (tumor node metastasis) and so on; overall survival of the patients showing high level of circPVT1 in tumor was significantly shorter than those with low level of circPVT1. ${ }^{54}$ Thus, in NSCLC, circPVT1 seemed to be a novel and promising biomarker associated with disease progression and prognosis. NSCLC is an aggressive type of cancer showing poor sensitivity to chemotherapy or radiotherapy and with shorter overall life expectancy. ${ }^{75}$ CircPVT1 knockdown was shown to inhibit proliferation and induce apoptosis in NSCLC cell line; the finding was validated in primary tumor derived from the cell line on nude mice. ${ }^{54}$ Thus, evidences of in vitro and in vivo experiments raised the possibility of novel therapeutics targeting circPVT1 in tumor with an aim to improve overall survival of patients with NSCLC significantly.

\section{Osteosarcoma}

In OS, serum circPVT1 level was established as a suitable diagnostic marker and moreover it was found to be more sensitive than conventional biomarker like alkaline phosphatase (ALP). ${ }^{53}$ Significant association of circPVT1 with tumors of advanced clinical stage and with poor patient outcome indicated prognostic importance of the candidate. In OS cell lines showing resistance to cisplatin and doxorubicin, circPVT1 level was significantly higher than those sensitive to the drugs; siRNA-mediated knockdown of circPVT1 was shown to reverse chemoresistance property of OS cell lines, and this might be through downregulation of multidrug transporter, ABCB1. ${ }^{53}$ Thus, in OS, circPVT1 level may be used to assess drug responsiveness of tumor; moreover, in overexpressing circPVT1 OS, circPVT1 may be targeted by novel therapeutics to modulate such response in favour of better patient outcome.

\section{ONCOGENIC NON-CODING RNA: CIRCPVT1 VERSUS LNCPVT1}

Although circPVT1 and lncPVT1 are derived from the same genetic locus, circPVT1 seemed to be more important as an oncogenic non-coding RNA over its linear counterpart.

1. CircPVT1 was found to upregulate its own transcription in cooperation with mutant $\mathrm{p} 53$, a protein frequently overexpressed during tumorigenesis ${ }^{15}$; therefore, overexpression of circPVT1 seemed to be more frequent in tumor than that of lncPVT1 in which such transcriptional regulation was not reported.

2. CircPVT1 is resistant to exonuclease digestion and therefore more stable with higher half-life than $\operatorname{lncPVT} 11^{14}$; so in blood and other body fluids of diseased (malignancy) individuals, 
circPVT1 level might serve as a more sensitive biomarker for diagnostic and prognostic purposes than that of its linear counterpart.

3. CircPVT1 and lncPVT1 served as competitive endogenous RNA to tumor-suppressive miRNAs and thereby causing upregulation of downstream oncogenic target. CircPVT1 is localised primarily in cytoplasm and hence seemed to regulate cytoplasm-centric miRNA-mRNA interaction more efficiently than that of lncPVT1 which is primarily nuclear localised. ${ }^{14}$

4. Due to its circular shape, circPVT1 did not seem to be degraded following binding with miRNA ${ }^{14}$; therefore, it might serve as more potent miRNA sponge than that of IncPVT1 which was linear and hence more prone to degradation following interaction with miRNA.

\section{CONCLUSION}

CircPVT1 remained largely unexplored in comparison with its linear counterpart lncPVT1. Although circPVT1 has been studied in few malignancies, more detailed studies in different malignant conditions as well as in other abnormalities remain unexplored.

Functionally, circPVT1 was reported to act as miRNA sponge, that is, circPVT1 binds to different miRNAs competitively, majority of which belong to tumor suppressive category and hence can suppress translation of oncogenic proteins like MKI67, ${ }^{15} \mathrm{c}-\mathrm{Myc},{ }^{28} \mathrm{Bcl}-2,{ }^{54} \mathrm{ABCB} 1^{53}$ and so on. Thus, circPVT1, by its competitive interaction with target miRNA and thereby attenuating their action, induces the production of oncogenic proteins as downstream effectors that seemed to promote tumor-associated phenotypes like proliferation, invasion, angiogenesis, drug resistance and so on. However, except serving as miRNA sponge, no other functional mechanism of the RNA has been known until the present. Recently, Tashiro et $a l^{76}$ reported potency of circPVT1 to encode a protein of 140 amino acids annotated as pvt 1 encoded protein; however, validation of such protein in a wet laboratory experiment with structural and functional details is still awaited. Therefore, considering huge oncogenic potency of circPVT1, an emerging star in cancer biology, further research work in all possible aspects is warranted for better understanding of the candidate as well as its contribution in associated disease pathogenesis.

\section{Take home message}

- CircPVT1 is an oncogenic circular RNA derived from exon 3 of IncPVT1 by back splicing.

- CircPVT1 serves as miRNA sponge to upregulate downstream oncogenic effectors and induces tumorigenesis.

- Overexpression of circPVT1 was shown to have diagnostic and prognostic importance in different malignancies.

- CircPVT1 level showed significant impact on drug response of tumors highlighting its therapeutic potential.

- CircPVT1 may be a promising target of novel therapeutics especially for drug-resistant tumors in the near future.

Correction notice This article has been corrected since it was published Online First. Figure 2 has been replaced to avoid duplication of the figure legend in the image.

Handling editor Professor Des Richardson.

Acknowledgements We are thankful to the Hon'ble Vice Chancellor, Presidency University Kolkata, India.
Contributors JA did data collection, data interpretation and wrote the manuscript. SC did study design. SD and SB did data collection, data interpretation and review of literature. $A D$ did data interpretation and wrote the manuscript. AG did study design, data interpretation, manuscript preparation and approved the final version of the manuscript.

Funding Financial support for this work was provided by Department of Higher Education Science and Technology and Biotechnology (793(Sanc.)/ST/P/ S\&T/1G-2/2016, Dt. 06/12/2016) to AG.

Competing interests None declared.

Patient consent for publication Not required.

Provenance and peer review Not commissioned; externally peer reviewed.

\section{REFERENCES}

1 Han Li C, Chen Y, Small CY. Small and long non-coding RNAs: novel targets in perspective cancer therapy. Curr Genomics 2015;16:319-26.

2 Ponting $\mathrm{CP}$, Oliver PL, Reik W. Evolution and functions of long noncoding RNAs. Cell 2009;136:629-41.

3 Huang B, Zhang R. Regulatory non-coding RNAs: revolutionizing the RNA world. Mol Biol Rep 2014;41:3915-23.

4 Chen L-L, Yang L. Regulation of circRNA biogenesis. RNA Biol 2015;12:381-8.

5 Tseng Y-Y, Moriarity BS, Gong W, et al. PVT1 dependence in cancer with Myc copynumber increase. Nature 2014;512:82-6.

6 Guan Y, Kuo W-L, Stilwell JL, et al. Amplification of PVT1 contributes to the pathophysiology of ovarian and breast cancer. Clin Cancer Res 2007;13:5745-55.

7 Wang F, Yuan J-H, Wang S-B, et al. Oncofetal long noncoding RNA PVT1 promotes proliferation and stem cell-like property of hepatocellular carcinoma cells by stabilizing NOP2. Hepatology 2014;60:1278-90.

8 Zhou Q, Chen F, Zhao J, et al. Long non-coding RNA PVT1 promotes osteosarcoma development by acting as a molecular sponge to regulate miR-195. Oncotarget 2016;7:82620-8263.

9 Zou H, Wu L-X, Yang Y, et al. IncRNAs PVT1 and HAR1A are prognosis biomarkers and indicate therapy outcome for diffuse glioma patients. Oncotarget 2017;8:78767-80.

10 Posa I, Carvalho S, Tavares J, et al. A pan-cancer analysis of MYC-PVT1 reveals CNV-unmediated deregulation and poor prognosis in renal carcinoma. Oncotarget 2016;7:47033-41.

11 Yuan CL, Li H, Zhu L, et al. Aberrant expression of long noncoding RNA PVT1 and its diagnostic and prognostic significance in patients with gastric cancer. Neoplasma 2016:63:442-9.

12 Cui D, Yu C-H, Liu M, et al. Long non-coding RNA PVT1 as a novel biomarker for diagnosis and prognosis of non-small cell lung cancer. Tumor Biol. 2016;37:4127-34.

13 Panda AC, Grammatikakis I, Kim KM, et al. Identification of senescence-associated circular RNAs (SAC-RNAs) reveals senescence suppressor CircPVT1. Nucleic Acids Res 2017a;45:4021-35.

14 Chen J, Li Y, Zheng Q, et al. Circular RNA profile identifies circPVT1 as a proliferative factor and prognostic marker in gastric cancer. Cancer Lett 2017;388:208-19.

15 Verduci L, Ferraiuolo M, Sacconi A, et al. The oncogenic role of circPVT1 in head and neck squamous cell carcinoma is mediated through the mutant p53/YAP/TEAD transcription-competent complex. Genome Biol 2017;18:1-24.

16 Xu M-D, Wang Y, Weng W, et al. A positive feedback loop of InCRNA-PVT1 and FOXM 1 facilitates gastric cancer growth and invasion. Clin Cancer Res 2017;23:2071-80.

17 Li X, Yang L, Chen L-L. The biogenesis, functions, and challenges of circular RNAs. Mol Cell 2018;71:428-42.

18 Quan G, Li J. Circular RNAs: biogenesis, expression and their potential roles in reproduction. J Ovarian Res 2018;11:1-12.

19 Wang F, Nazarali AJ, Ji S. Circular RNAs as potential biomarkers for cancer diagnosis and therapy. Am J Cancer Res 2016;6:1167-76.

20 Northcott PA, Shih DJH, Peacock J, et al. Subgroup-specific structural variation across 1,000 medulloblastoma genomes. Nature 2012;488:49-56.

21 Ferber MJ, Eilers P, Schuuring E, et al. Positioning of cervical carcinoma and Burkitt lymphoma translocation breakpoints with respect to the human papillomavirus integration cluster in FRA8C at 8q24.13. Cancer Genet Cytogenet 2004;154:1-9.

22 Bønnelykke K, Matheson MC, Pers TH, et al. Meta-analysis of genome-wide association studies identifies ten loci influencing allergic sensitization. Nat Genet 2013:45:902-6.

23 Yu B, Zheng Y, Alexander D, et al. Genome-wide association study of a heart failure related metabolomic profile among African Americans in the Atherosclerosis Risk in Communities (ARIC) study. Genet Epidemiol 2013;37:840-5.

24 Wasserman NF, Aneas I, Nobrega MA. An 8q24 gene desert variant associated with prostate cancer risk confers differential in vivo activity to a Myc enhancer. Genome Res 2010;20:1191-7.

25 Ghoussaini $M$, Song $H$, Koessler T, et al. Multiple loci with different cancer specificities within the 8q24 gene desert. J Natl Cancer Inst 2008;100:962-6.

26 Meyer KB, Maia A-T, O’Reilly M, et al. A functional variant at a prostate cancer predisposition locus at $8 \mathrm{q} 24$ is associated with PVT1 expression. PLoS Genet 2011;7:e1002165. 
27 Carramusa L, Contino F, Ferro A, et al. The pvt-1 oncogene is a MYC protein target that is overexpressed in transformed cells. J Cell Physiol 2007;213:511-8.

$28 \mathrm{Hu}$ J, Han Q, Gu Y, et al. Circular RNA PVT1 expression and its roles in acute lymphoblastic leukemia. Epigenomics 2018;10:723-32.

29 Zhang Y, Zhang X-O, Chen T, et al. Circular intronic long noncoding RNAs. Mol Cell 2013:51:792-806.

30 Jeck WR, Sorrentino JA, Wang K, et al. Circular RNAs are abundant, conserved, and associated with Alu repeats. RNA 2013;19:141-57.

31 Li Z, Huang C, Bao C, et al. Exon-intron circular RNAs regulate transcription in the nucleus. Nat Struct Mol Biol 2015a;22:256-64.

32 Cocquerelle C, Mascrez B, Hétuin D, et al. Mis-splicing yields circular RNA molecules. FASEB J 1993;7:155-60.

33 Qian L, Vu MN, Carter M, et al. A spliced intron accumulates as a lariat in the nucleus of T cells. Nucleic Acids Res 1992:20:5345-50.

34 Salzman J, Gawad C, Wang PL, et al. Circular RNAs are the predominant transcript isoform from hundreds of human genes in diverse cell types. PLoS One 2012; 7:e30733

35 Jeck WR, Sharpless NE. Detecting and characterizing circular RNAs. Nat Biotechnol 2014;32:453-61.

36 Zhang $\mathrm{X}-\mathrm{O}$, Wang $\mathrm{H}-\mathrm{B}$, Zhang $\mathrm{Y}$, et al. Complementary sequence-mediated exon circularization. Cell 2014;159:134-47.

37 Ashwal-Fluss R, Meyer M, Pamudurti NR, et al. circRNA biogenesis competes with pre-mRNA splicing. Mol Cell 2014;56:55-66.

38 Conn SJ, Pillman KA, Toubia J, et al. The RNA binding protein quaking regulates formation of circRNAs. Cell 2015;160:1125-34.

39 Wilusz JE, Sharp PA. A circuitous route to noncoding RNA. Science 2013;340:440-1.

40 Starke S, Jost I, Rossbach 0, et al. Exon circularization requires canonical splice signals. Cell Rep 2015;10:103-11.

41 Salzman J, Chen RE, Olsen MN, et al. Cell-type specific features of circular RNA expression. PLoS Genet 2013;9:e1003777.

42 Ghosh S, Ghosh A, Maiti GP, et al. Alterations of ROBO1/DUTT1 and Robo2 loci in early dysplastic lesions of head and neck: clinical and prognostic implications. Hum Genet 2009;125:189-98.

43 Mitra S, Mazumder-Indra D, Mondal RK, et al. Inactivation of SLIT2-ROBO1/2 pathway in premalignant lesions of uterine cervix: clinical and prognostic significances. PLoS One 2012;7:e38342.

44 Li J-H, Liu S, Zheng L-L, et al. Discovery of protein-IncRNA interactions by integrating large-scale CLIP-Seq and RNA-seq datasets. Front Bioeng Biotechnol 2014;2:88.

45 Lubelsky Y, Ulitsky I. Sequences enriched in Alu repeats drive nuclear localization of long RNAs in human cells. Nature 2018;555:107-11.

46 Arnberg AC, Van Ommen GJ, Grivell LA, et al. Some yeast mitochondrial RNAs are circular. Cell 1980;19:313-9.

47 Suzuki H, Zuo Y, Wang J, et al. Characterization of RNase R-digested cellular RNA source that consists of lariat and circular RNAs from pre-mRNA splicing. Nucleic Acids Res 2006;34:e63.

48 Panda AC, De S, Grammatikakis I, et al. High-purity circular RNA isolation method (RPAD) reveals vast collection of intronic circRNAs. Nucleic Acids Res 2017b;45:e116.

49 Zhang X-O, Dong R, Zhang Y, et al. Diverse alternative back-splicing and alternative splicing landscape of circular RNAs. Genome Res 2016a;26:1277-87.

50 Zhang Y, Yang L, Chen L, et al. Long non-coding RNAs. Methods Mol Biol 2016:1402:215-27.

51 Dudekula DB, Panda AC, Grammatikakis I, et al. Circlnteractome: a web tool for exploring circular RNAs and their interacting proteins and microRNAs. RNA Biol 2016;13:34-42.

52 Schneider T, Schreiner S, Preußer C, et al. Northern blot analysis of circular RNAs. Methods Mol Biol 1724;2018:119-33.

53 Kun-Peng Z, Xiao-Long M, Chun-Lin Z. Overexpressed circPVT1, a potential new circular RNA biomarker, contributes to doxorubicin and cisplatin resistance of osteosarcoma cells by regulating ABCB1. Int J Biol Sci 2018;14:321-30.
54 Qin S, Zhao Y, Lim G, et al. Circular RNA PVT1 acts as a competing endogenous RNA for miR-497 in promoting non-small cell lung cancer progression. Biomed Pharmacother 2019;111:244-50

55 Huang T, Liu HW, Chen JQ, et al. The long noncoding RNA PVT1 functions as a competing endogenous RNA by sponging miR-186 in gastric cancer. Biomed Pharmacother 2017;88:302-8.

56 Zhao J, Du P, Cui P, et al. LncRNA PVT1 promotes angiogenesis via activating the STAT3/VEGFA axis in gastric cancer. Oncogene 2018;37:4094-109.

57 Li T, Meng X-L, Yang W-Q. Long noncoding RNA PVT1 acts as a "sponge" to inhibit microRNA-152 in gastric cancer cells. Dig Dis Sci 2017;62:3021-8.

58 Yu C, Wang Y, Li G, et al. LncRNA PVT1 promotes malignant progression in squamous cell carcinoma of the head and neck. J Cancer 2018;9:3593-602.

59 Wu J, Yang L, Shan Y, et al. AURKA promotes cell migration and invasion of head and neck squamous cell carcinoma through regulation of the AURKA/Akt/FAK signaling pathway. Oncol Lett 2016;11:1889-94.

60 Zhi X, Lamperska K, Golusinski P, et al. Gene expression analysis of head and neck squamous cell carcinoma survival and recurrence. Oncotarget 2015;6:547-55.

61 Ohshima K, Haraoka S, Yoshioka S, et al. Mutation analysis of mitotic checkpoint genes (hBUB1 and hBUBR1) and microsatellite instability in adult T-cell leukemia/ lymphoma. Cancer Lett 2000;158:141-50.

62 Shichiri M, Yoshinaga K, Hisatomi H, et al. Genetic and epigenetic inactivation of mitotic checkpoint genes hBUB1 and hBUBR1 and their relationship to survival. Cancer Res 2002:62:13-17.

63 Wong T-S, Man O-Y, Tsang C-M, et al. MicroRNA let-7 suppresses nasopharyngeal carcinoma cells proliferation through downregulating c-myc expression. J Cancer Res Clin Oncol 2011;137:415-22.

64 Zhou M, Liu Z, Zhao Y, et al. MicroRNA-125b confers the resistance of breast cancer cells to paclitaxel through suppression of pro-apoptotic Bcl-2 antagonist killer 1 (BAK1) expression. J Biol Chem 2010;285:21496-507.

$65 \mathrm{Mi}$, Lu J, Sun M, et al. MicroRNA expression signatures accurately discriminate acute lymphoblastic leukemia from acute myeloid leukemia. Proc Natl Acad Sci U SA 2007; 104:19971-6.

66 Tassano E, Acquila M, Tavella E, et al. MicroRNA-125b-1 and BLID upregulation resulting from a novel $\mathrm{IgH}$ translocation in childhood B-cell precursor acute lymphoblastic leukemia. Genes Chromosomes Cancer 2010;49:682-7.

67 Yang Y-R, Zang S-Z, Zhong C-L, et al. Increased expression of the IncRNA PVT1 promotes tumorigenesis in non-small cell lung cancer. Int I Clin Exp Pathol 2014; 7:6929-35

68 Zhao WY, Wang Y, An ZJ, et al. Downregulation of miR-497 promotes tumor growth and angiogenesis by targeting HDGF in non-small cell lung cancer. Biochem Biophys Res Commun 2013;435:466-71.

69 Guo D, Wang Y, Ren K, et al. Knockdown of LncRNA PVT1 inhibits tumorigenesis in non-small-cell lung cancer by regulating miR-497 expression. Exp Cell Res 2018;362:172-9.

70 Song J, Wu X, Liu F, et al. Long non-coding RNA PVT1 promotes glycolysis and tumor progression by regulating miR-497/HK2 axis in osteosarcoma. Biochem Biophys Res Commun 2017;490:217-24.

71 Sun Z-Y, Jian Y-K, Zhu H-Y, et al. IncRNA PVT1 targets miR-152 to enhance chemoresistance of osteosarcoma to gemcitabine through activating c-MET/PI3K/AKT pathway. Pathol Res Pract 2019;215:555-63.

72 Lee $\mathrm{Y}-\mathrm{H}$, Yang H-W, Yang L-C, et al. DHFR and MDR1 upregulation is associated with chemoresistance in osteosarcoma stem-like cells. Oncol Lett 2017:14:171-9.

73 Zou Z, Zou R, Zong D, et al. miR-495 sensitizes MDR cancer cells to the combination of doxorubicin and taxol by inhibiting MDR1 expression. J Cell Mol Med 2017:21:1929-43.

74 Misra S, Ghatak S, Toole BP. Regulation of MDR1 expression and drug resistance by a positive feedback loop involving hyaluronan, phosphoinositide 3-kinase, and ErbB2. J Biol Chem 2005;280:20310-5.

75 Pastorino U. Lung cancer screening. Br J Cancer 2010;102:1681-6.

76 Tashiro K, Tseng Y-Y, Konety B, et al. MP99-18 role of long non-coding RNA PVT1 in regulating MYC in human cancer. J Urol 2017;197:e1327-8. 